fournal of Medical Genetics (1974). 11, 91.

\section{Arthrogryposis Multiplex Congenita: Neurogenic Type with Autosomal Recessive Inheritance}

\author{
Summary. An infant affected by \\ severe arthrogryposis multiplex congenita \\ leading to death in infancy due to neuro- \\ genic atrophy is described. Six other \\ sibs were similarly affected. An auto- \\ somal recessive mode of inheritance is \\ suggested.
}

Arthrogryposis multiplex congenita (AMC) is characterized by congenital immobility of the limbs with fixation of multiple joints in certain postures and muscle wasting. This condition may be caused by neurogenic muscular atrophy (Frischknecht, Bianchi, and Pilleri, 1960; Bargeton et al, 1961; Drachman and Banker, 1961 ; Peña et al, 1968) or by primary muscular dystrophy (Banker, Vitcor, and Adams, 1957; Srivastava, 1968) as well as by other aetiologies (Ek, 1958; Bargeton et al, 1961; Pearson and Fowler, 1963; Hooshmand, Martinez, and Rosenblum, 1971). Although most cases described were sporadic (Banker et al, 1957; Ek, 1958; Friedlander, Westin, and Wood, 1968) familial hereditary cases have also been observed (Bargeton et al, 1961; Pearson and Fowler, 1963; Lebenthal et al, 1970).

Hereditary cases of AMC due to neurogenic muscular atrophy were reported to be transmitted by an autosomal dominant gene (Swinyard, 1960; Lebenthal et al, 1970) although a few families were reported in whom autosomal recessive inheritance was suggested (Frischknecht et al, 1960; Peña et al, 1968). AMC due to primary muscular dystrophy behaves as an autosomal recessive trait (Friedlander et al, 1968; Lebenthal et al, 1970).

This communication describes sibs of one family affected by severe arthrogryposis multiplex congenita with underlying advanced neurogenic muscu-

Received 14 August 1973. lar atrophy, in which the mode of inheritance appears to be autosomal recessive.

\section{Case Report}

B.L., a 12-day-old female, was hospitalized with severe postural anomalies of the limbs. She was the product of a normal pregnancy, except for the lack of fetal movements since the sixth month of pregnancy. At birth which was in breech presentation, the infant weighed $3000 \mathrm{~g}$ and was cyanotic. The cyanosis disappeared during the stay of 2 days in an incubator.

On admission she weighed $2950 \mathrm{~g}$. Physical examination revealed a generalized moderate hypertrichosis, nonsymmetrical low set ears, a high arched palate, and a funnel chest. Both shoulders were dislocated, the right upper limb manifested flaccid paralysis with a slight ulnar deviation of the hand. The left elbow was flexed and the hand was fixed in dorsiflexion. The large joints of both legs were also fixed in flexion (Fig. 1). Severe

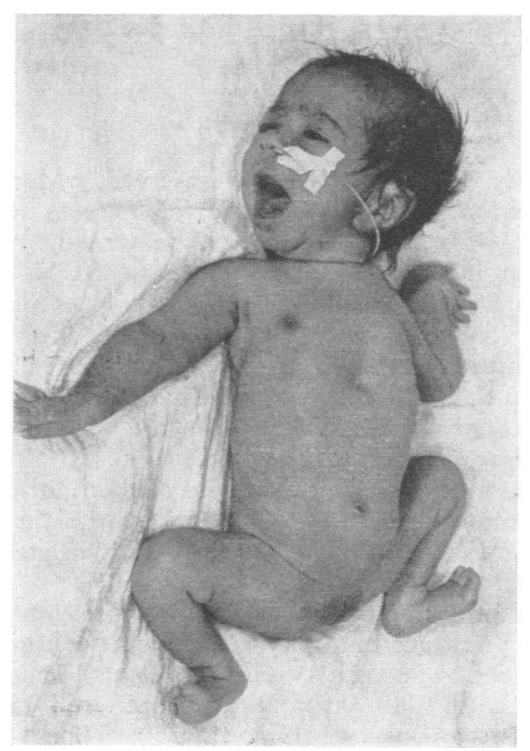

FIG. 1. The proposita at the age of 1 month. 


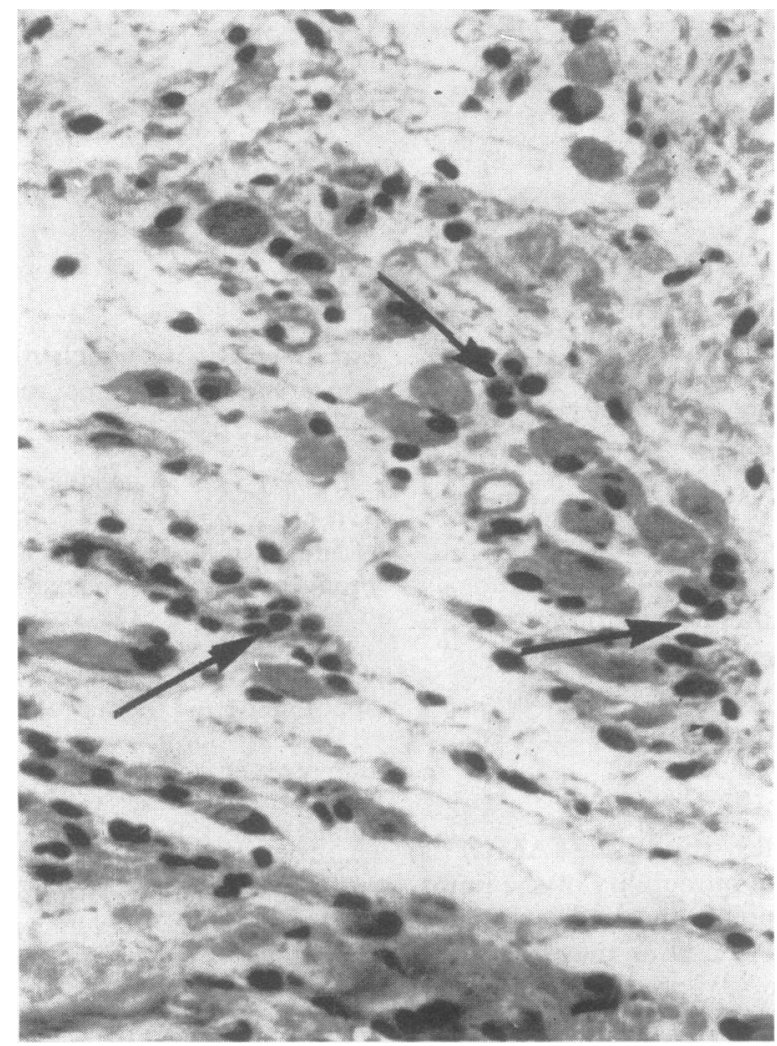

FIg. 2. Histological picture of right gastrocnemius muscle. Arrows indicate groups of small fibres. (H \& E, $\times 420$.)

generalized muscle wastage was observed. Moro, tonic neck, tendon, abdominal, and grasp reflexes were not elicited. Only the sucking reflex was normal.

Haematological examination showed BUN, electrolytes, $\mathrm{Ca}, \mathrm{P}$, uric acid, and liver function tests all to be normal. Mucopolysaccharide spot test, Kahn, Wassermann, and urine chromatography for amino acids were all negative. Pertinent enzyme studies showed aldolase $=24.8 \mathrm{u}$ (normal range: $15 \cdot 5-17.5 \mathrm{u}$ ). Creatine phosphokinase $=25 \cdot 0 \mathrm{u}$ (normal range : $6-50 \mathrm{u}$ ). EMG revealed absence of electrical activity of the right brachioradialis muscle and low activity of the right tibialis anterior. IVP, GIT, skull, chest, and skeletal $x$-rays were normal as was EEG.

Biopsy of the right gastrocnemius muscle showed an extremely advanced atrophy with extensive fibrosis and replacement by fat tissue. Groups of small fibres representing motor unit atrophy were scattered randomly within dense connective tissue (Fig. 2). These features are compatible with an advanced spinal type of muscle atrophy. Chromosomal study revealed a normal female

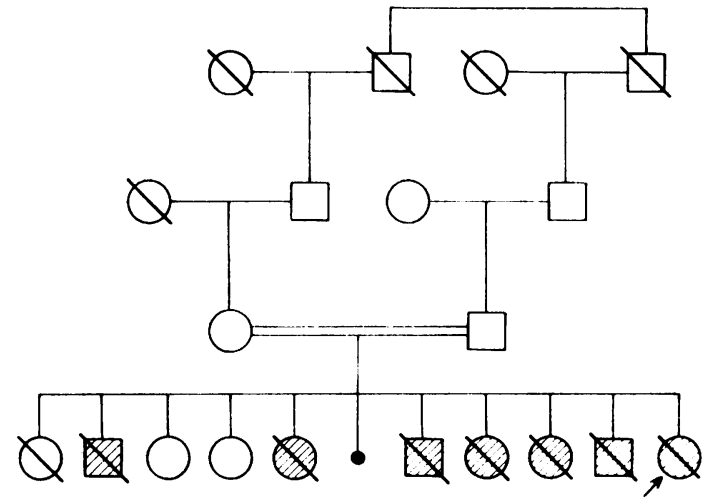

Da male, AFFECIED

FIG. 3. Family pedigree. 
karyotype. The baby died at 3 months of age from aspiration pneumonia. It was not possible to perform a necropsy.

\section{Family History}

In this family, the parents are second cousins (Fig. 3). The mother had 11 pregnancies and received penicillin for syphilis during the last two pregnancies. Of the 10 previous pregnancies one terminated in a miscarriage and three in stillbirths. Among six live births, four children were born affected with similar manifestations as the proposita (see Fig. 3). They were all affected by multiple joint flexions and funnel chests and died within 4 months of birth. According to the parents' description two of the stillbirths were similarly affected. Two daughters aged 16 and 14 years are healthy.

\section{Discussion}

In this family seven sibs were similarly affected by AMC; a muscle biopsy was performed only in the proposita. The severe muscle wasting being present at birth and histologically demonstrated, suggested that the disease process developed during fetal life. Moreover, the almost normal levels of serum aldolase and CPK indicated that no further destruction of muscle occurred after birth when the muscle mass was almost completely absent, as confirmed by the biopsy despite extreme alterations of the muscular components. The presence of groups of small muscle fibres supported the diagnosis of neurogenic muscular atrophy.

In most reports on familial neurogenic AMC the mode of inheritance was that of an autosomal dominant trait (Lebenthal et al, 1970) and the patients survived until late childhood (Kanof, Aronson, and Volk, 1956; Peña et al, 1968). Myopathic AMC, with a shorter life span, has been described in sibs as transmitted by an autosomal recessive gene (Friedlander et al, 1968; Peña $e t a l, 1968$; Lebenthal et al, 1970). Yet, most of the published cases of AMC are sporadic (Hooshmand et al, 1971).

Five of the affected sibs in the family described here died during early infancy and two were born dead. This short survival is contrary to that previously observed. The fact that sibs of both sexes were affected while both parents were healthy and consanguineously related suggests an autosomal recessive pattern of transmission. However, the fact that seven out of 10 sibs were affected does not seem to agree well with the expected ratio of autosomal recessive disease. Furthermore, the probability of obtaining such a family based on the recessive mode of inheritance is approximately $1 / 300$. Such an occurrence is rare but plausible, considering the selective nature of the patient material referred for genetic counselling. Addi- tionally, the fact that both males and females are affected, eliminates the possibility of a sex-linked recessive trait. Nonetheless, one cannot rule out the possibility of a dominant, autosomal, or sexlinked mutation in the germ cells of one of the parents or the mother, respectively. The most acceptable of the above alternatives seems to be that of autosomal recessive inheritance.

Polygenic inheritance was considered in this family, but the negative history among other members of the kindred, in spite of additional consanguineous marriages between spouses who had the same common ancestors as this family, argues against such a consideration. No environmental factors which may have been responsible for the syndrome were observed, but this possibility cannot be excluded.

There is some resemblance between this family and that described by Bargeton et al (1961). In the latter family the parents were healthy and first cousins and had three children. The two youngest children were affected with neurogenic type of AMC; one was stillborn and the youngest died immediately after birth. Consanguinity among parents, and severe neurogenic AMC in sibs with early death were the most important similarities observed in both families. Autosomal recessive inheritance is suggested in both families.

A further point of interest was the presence of funnel chest in the affected sibs only. This deformity, as an isolated malformation, is occasionally transmitted by an autosomal dominant gene (Lebenthal et al, 1970).

We wish to thank Dr T. Cohen and Professor M. M. Cohen for their critical evaluation of the manuscript; Professor A. Behar for the histological interpretation; and Dr E. Shapira for referring the case.

A. Rosenmann and I. Arad

Departments of Human Genetics and Pediatrics, Hadassah University Hospital, Ferusalem, Israel

\section{REFERENCES}

Banker, B. Q., Vitcor, M., and Adams, R. D. (1957). Arthrogryposis multiplex due to congenital muscular dystrophy. Brain, 80 , 319-334.

Bargeton, E., Nezelof, C., Guran, P., and Job, J. C. (1961). Étude anatomique d'un cas d'arthrogrypose multiple congénitale et familiale. Revue Neurologique, 104, 479-489.

Drachman, D. B. and Banker, B. Q. (1961). Arthrogryposis multiplex congenita: case due to disease of the anterior horn cells. Archives of Neurology, 5, 77-93.

Ek, J. I. (1958). Cerebral lesions in arthrogryposis multiplex congenita. Acta Paediatrica, 47, 302-316.

Friedlander, H. L., Westin, G. W., and Wood, W. L. (1968). Arthrogryposis multiplex congenita. A review of forty-five cases. fournal of Bone and foint Surgery, 50A, 89-112.

Frischknecht, V. W., Bianchi, L., and Pilleri, G. (1960). Familiäre arthogryposis multiplex congenita. Neuro-arthro-myodysplhasia congenita. Helvetica Paediatrica Acta, 15, 259-279. 
Hooshmand, H., Martinez, A. J., and Rosenblum, W. I. (1971) Arthrogryposis multiplex congenita: simultaneous involvement of peripheral nerve and skeletal muscle. Archives of Neurology, 24 , 561-572.

Kanof, A., Aronson, S. M., and Volk, B. W. (1956). Arthrogryposis: A clinical and pathological study of three cases. Pediatrics, 17, 532-540.

Lebenthal, E., Shochet, S. B., Adam, A., Seelenfreund, M., Fried, A., Najenson, T., Sandbank, U., and Matoth, Y. (1970). Arthrogryposis multiplex congenita: twenty three cases in an Arab kindred. Pediatrics, 46, 891-899.

Pearson, C. M. and Fowler, W. G., Jr. (1963). Hereditary nonprogressive muscular dystrophy inducing arthrogryposis syndroms. Brain, 86, 75-88.

Peña, C. E., Miller, F., Budzilovich, G. N., and Feigin, I. (1968). Arthrogryposis multiplex congenita: report of two cases of a radicular type with familial incidence. Neurology, 18, 926-930.

Srivastava, R. N. (1968). Arthrogryposis multiplex congenita: case report of two siblings. Clinical Pediatrics, 7, 691-694.

Swinyard, C. A. (1960). Progressive muscular dystrophy and atrophy and related conditions. Diagnosis and management. Pediatric Clinics of North America, 7, 703-732.

\section{A Case of Meningomyelocele in a Kindred with Multiple Cases of Spondylolisthesis and Spina Bifida Occulta*}

\author{
Summary. A child with meningo- \\ myelocele, and other spinal anomalies, \\ was born to a woman with three sibs who \\ had spondylolisthesis and whose father \\ and two of his sibs had a history of back \\ problems. The suggestion is made that \\ these anomalies represent a pattern of \\ autosomal dominant inheritance with \\ variable expressivity.
}

In an epidemiological study of spina bifida cystica a proposita with a strong maternal family history of spondylolisthesis was identified. Although spina bifida occulta is commonly found associated with spondylolisthesis, this family is of interest because of the presence of a case of meningomyelocele (the proposita) in a family with a marked occurrence of spondylolisthesis and spina bifida occulta.

\section{Case Reports}

The Proposita (IV.1) was admitted to The Children's Orthopedic Hospital and Medical Center in 1972, at the

Received 30 July 1973.

* This work was supported in part by NIH grant GM 15253. age of 2 days, for evaluation and treatment of an epithelialized $2 \times 4 \mathrm{~cm}$ cystic mass at T-4. The child was the product of a normal pregnancy and delivery to a 19-year-old, para 0 , white female. Radiology and myelography showed: (1) hemivertebrae of T-10 and also $\mathrm{T}-3$ through $\mathrm{T}-6$ or $\mathrm{T}-7 ;$ (2) deformed ribs; and (3) diastematomyelia at $\mathrm{L}-1$. The cystic mass was found at surgery to be a T-4 meningomyelocele. When the mother was later contacted as part of an epidemiological study of spina bifida cystica she informed us that three of her older sibs had been treated at University Hospital for a 'back defect'. These charts were reviewed and an additional sib found who had been evaluated for low back pain at the University Hospital. There was no family history of spina bifida cystica, anencephalus, or hydrocephalus.

K.T. (III.1) was seen in 1969 at University Hospital at age 22. She had had an onset of inconstant low back pain 2 years previously which had recurred. Radiological examination showed a bilateral defect in the pars interarticularis of L-5 with grade II spondylolisthesis at L5-S1. In addition spina bifida occulta of L-5 was demonstrated. She was treated by L-5 laminectomy and L5-S1 discectomy.

R.L.S. (III.2) was seen at University Hospital in 1968 at age 20 with a history of low back pain for many years. Upon $x$-ray examination the patient was found to have spondylolysis with anterior displacement of L-5 under L-4 (reverse spondylolisthesis), bilateral defects of the pars interarticularis, and slight spina bifida occulta of L-5. Treatment consisted of L-5 hemilaminectomy (rt) and L5-S1 and L4-5 discectomy.

J.L.S. (III.3) was seen at University Hospital, also in 1968 , at age 19 years, with a history of recurrent low back pain during the preceding 5 to 6 years. A myelogram revealed multiple anomalies of the L5-S1 area including bilateral defects in the pars interarticularis of L-5, grade I spondylolisthesis of L5-S1, and spina bifida occulta of S-1. The patient was treated by L-5 laminectomy with L5-S1 discectomy.

D.S. (III.4) was seen in 1968 at University Hospital at age 17. He had a history of intermittent low back pain with onset at age 14. Radiology showed sacral erosion of the anterior portion of the spinal canal approximately $3 \mathrm{~cm}$ in size. Spina bifida occulta of L-5 was present while spondylolisthesis was not. A myelogram showed blunting of L-5 and S-1 nerve roots on the left. No treatment was carried out.

Family History. From the histories of patients K.T., R.L.S., J.L.S., and D.S. it was learned that there was a history of back problems in generation II of this family. Their father (II.6) had had back trouble since age 17. Two of his sibs (II.3 and II.4) also had histories of back problems. Thus, it appears that there is a 